\title{
Design of biomass value chains that are synergistic with the food-energy-water nexus: strategies and opportunities
}

\begin{abstract}
Humanity's future sustainable supply of energy, fuels and materials is aiming towards renewable sources such as biomass. Several studies on biomass value chains (BVCs) have demonstrated the feasibility of biomass in replacing fossil fuels. However, many of the activities along the chain can disrupt the food-energy-water (FEW) nexus given that these resource systems have been ever more interlinked due to increased global population and urbanisation. Essentially, the design of BVCs has to integrate the systems-thinking approach of the FEW nexus; such that, existing concerns on food, water and energy security, as well as the interactions of the BVCs with the nexus, can be incorporated in future policies. To date, there has been little to no literature that captures the synergistic opportunities between BVCs and the FEW nexus. This paper presents the first survey of process systems engineering approaches for the design of BVCs, focusing on whether and how these approaches considered synergies with the FEW nexus. Among the surveyed mathematical models, the approaches include multi-stage supply chain, temporal and spatial integration, multi-objective optimisation and uncertainty-based risk management. Although the majority of current studies are more focused on the economic impacts of BVCs, the mathematical tools can be remarkably useful in addressing critical sustainability issues in BVCs. Thus, future research directions must capture the details of food-energy-water interactions with the BVCs, together with the development of more insightful multi-scale, multi-stage, multi-objective and uncertainty-based approaches.
\end{abstract}

Keyword: Biomass value chains (BVCs); Food-energy-water (FEW) nexus; Mathematical modelling; Biomass supply chains; Optimisation; Process systems engineering; Sustainable land use; Bioenergy 